\title{
The geometric reason for the non-existence of a $\mathrm{MOL}(6)$
}

\section{Dieter Betten ${ }^{1}$}

Received: 30 March 2021 / Accepted: 7 December 2021 / Published online: 7 January 2022

(c) The Author(s) 2022

\begin{abstract}
The problem of Euler concerning the 36 officers, (Euler, in Leonardi Euleri Opera Ser I 7:291-392, 1782), was first solved by Tarry (Comptes rendus Ass Franc Sci Nat 1 (1900) 2:170-203, 1901). Short proofs for the non-existence were given in Betten (Unterricht 36:449-453, 1983), Beth et al. (Design Theory, Bibl. Inst. Mannheim, Wien, Zürich, 1985), Stinson (J Comb Theory A 36:373-376, 1984). This problem is equivalent to the existence of a $\operatorname{MOL}(6)$, i. e., a pair of mutually orthogonal latin squares of order 6. Therefore in Betten (Mitt Math Ges Hamburg 39:59-76, 2019; Res Math 76:9, 2021; Algebra Geom 62:815-821, 2021) the structure of a (hypothetical) MOL(6) was studied. Now we combine the old proofs and the new studies and filter out a simple way for the proof of non-existence. The aim is not only to give still other short proofs, but to analyse the problem and reveal the geometric reason for the non-existence of a MOL(6)- and the non-solvability of Euler's problem.
\end{abstract}

Keywords Orthogonal arrays · Latin squares - Room squares · Orthogonal designs · Configurations

\section{Mathematics Subject Classification O5 - S1}

\section{Introduction}

We recall Euler's 36 officers Problem: Can 36 officers, drawn from 6 different ranks and also from 6 different regiments, be ranged in a square so that in each line (both horizontal and vertical) there are 6 officers of different rank and different regiments? This was a famous problem at Euler's time, and Euler wrote a paper concerning it, (Euler 1782). The first proof of the non-existence was by Tarry (1901), so we may call this problem now the Euler/Tarry problem. The problem can be formulated in mathematical terms by the existence of a MOL(6) (a pair of mutually orthogonal latin squares LQ(6) of order 6), (Colbourne and Dinitz 1996; Denes and Keedwell 1974).

\footnotetext{
$\bowtie$ Dieter Betten

reteid@math.uni-kiel.de

1 Department of Mathematics, Christian-Albrechts-Universität Kiel, Heinrich-Hecht-Platz 6, Kiel 24118, Germany
} 
A latin square $\mathrm{LQ}(6)$ is an $(6 \times 6)$-array of numbers $1,2, \ldots, 6$, such that in each row and in each column of the array there are 6 different numbers. We call these numbers the digits. A transversal of the latin square LQ(6) is a 6-tuple of elements which meets each row and each column exactly once and has 6 different digits. If there are six pairwise disjoint transversals, we denote them by $t=1, t=2, \ldots, t=6$ (in some order), and the $6 \times 6$-array with these transversals is a second latin square of order 6 , called orthogonal to the first one. Both latin squares are mutually orthogonal and define a so called MOL(6).

The MOL(6) has 6 rows, 6 columns, 6 digits and 6 transversals, and defines a linear space on 24 variables, which we call LSP(24). It has 4 6-blocks and 36 4-blocks and each pair of variables is in exactly one block. So, a MOL(6) and a LSP(24) are structurally the same, only in different presentation.

In Sect. 2 we derive a tactical decomposition TDOII for the linear space LSP(24). This quotient structure for $\operatorname{LSP}(24)$ is also described for the MOL(6) and is the main notion in this paper. In Sect. 3 we study the decomposition TDOII. It has an outstanding and unique substructure Q, (Theorem 4), which can be seen dually as a quadruple of parallel classes on a set of 6 elements. In Theorem 3 an invariant $\mu_{4}$ is defined and it is shown $\mu_{4}=6$. This maximal number 6 is exactly reached by the 6 elements of $Q$. In Sect. 4 we prove that the quotient structure TDO II cannot be extended to a MOL(6). For this we choose various subsets of TDO II containing Q and derive in each case a contradiction. We also study the four latin squares contained in a MOL(6) and show that one of them does not exist. So we get several proofs for the non-existence of a MOL(6), but we applied computer methods.

To avoid this we start in Sect. 7 with two partial substructures of a MOL(6) and derive TDO II and prove $\mu_{4}=6$ without computer. Thus we get computer free proofs for the non-solvability of Euler's problem.

In Sect. 8 we prove that the automorphism group of a MOL(6) is the symmetric group $S_{4}$. This leads again to a non-existence proof for a MOL(6).

\section{A quotient structure for a MOL(6)}

We give an example of a MOL(6):

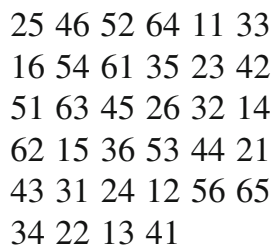

In each cell of the $(6 \times 6)$-array, there is a pair of numbers. We call the first number the digit (corresponding to the rank) and the second number the transversal (corresponding to the regiment). In the last row we had to let two gaps since the problem is not solvable. 
The $(6 \times 6)$-array of digits defines a latin square LQ $(6)$ of order 6 and the $(6 \times 6)$-array of transversals is a second latin square. A solution of Euler's problem means that each of the 36 combinations digit/transversal occurs exactly once, we say the two latin squares are mutually orthogonal.

We define a quotient structure of a MOL(6) by splitting the six numbers $\{1,2,3,4,5,6\}$ into two parts: the low numbers $\{1,2,3\}$ and the high numbers $\{4,5,6\}$ : So we define low digits $d \in\{1,2,3\}$ and high digits $H \in\{4,5,6\}$ and low transversals $t \in\{1,2,3\}$ and high transversals $T \in\{4,5,6\}$. Instead of 36 entries there are only 4 types of entries : $A=d t, B=d T, C=H t, D=H T$, each occuring 9 times. For the given example this leads to the following quotient structure:

\begin{tabular}{|llllll|}
\hline$B$ & $D$ & $C$ & $D$ & $A$ & $A$ \\
$B$ & $D$ & $C$ & $B$ & $A$ & $C$ \\
$C$ & $C$ & $D$ & $B$ & $A$ & $B$ \\
$C$ & $B$ & $B$ & $C$ & $D$ & $A$ \\
$C$ & $A$ & $B$ & $A$ & $D$ & $D$ \\
$B$ & $A$ & $A$ & $C$ & $D$ & $D$ \\
\hline
\end{tabular}

For a LSP(24) the tactical decompositions can be seen as quotient structures.

Theorem 1 Each MOL(6) and the corresponding LSP(24) admit the following quotient structures:

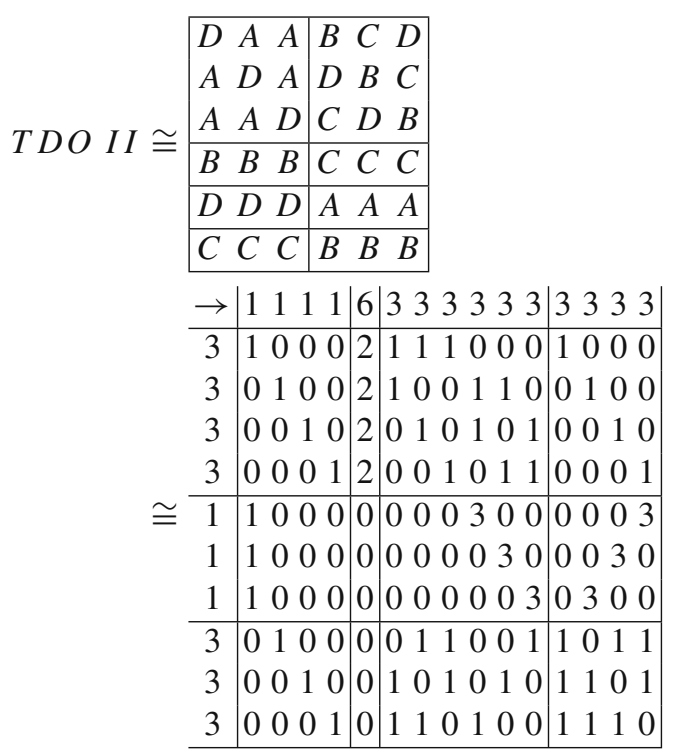


Proof In Betten (2019) we showed that each LSP(24) admits one of the following tactical decompositions:

$$
\begin{aligned}
& \begin{array}{l}
\rightarrow 111163333333333 \\
\hline 3 \mid 100021111000000
\end{array} \\
& \text { 3| } 01002100001110000 \\
& \text { 3| } 0010200100100110 \\
& \text { TDOI: } \frac{3 \mid 000120010010101}{3 \mid 100000000111111} \\
& \text { 3| } 0100000011100011 \\
& \text { 3| } 000100010011011001 \\
& \text { 3| } 0000101101101010 \\
& \begin{array}{l}
\rightarrow 1111163333333333 \\
\hline 3 \mid 1000021111000000
\end{array} \\
& \text { 3| } 010100021000111000 \\
& \text { 3| } 00102201000100110
\end{aligned}
$$

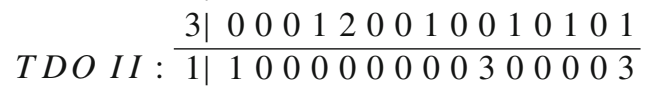

$$
\begin{aligned}
& \text { 1| } 1000000000030030 \\
& \begin{array}{l|lllllllllllllll}
1 \mid & 1 & 0 & 0 & 0 & 0 & 0 & 0 & 0 & 0 & 0 & 0 & 3 & 3 & 0 & 0 \\
\hline 3 \mid & 0 & 100000 & 0 & 1 & 1 & 10000 & 0 & 1 & 11
\end{array} \\
& \text { 3| } 000100100110011001 \\
& \text { 3| } 0000101101101010
\end{aligned}
$$

This are the computer outputs and the entries are the number of flags in the respective subrectangles. Using the notations $A=d t, B=d T, C=H t, D=H T$ we get the $(6 \times 6)$-arrays

$$
\left.T D O I \cong \begin{array}{lll|lll}
D & A & A & B & C & D \\
A & D & A & D & B & C \\
A & A & D & C & D & B \\
\hline D & B & C & A & B & C \\
C & D & B & C & A & B \\
B & C & D & B & C & A \\
\hline
\end{array}\right] T D O \quad I I \cong \begin{array}{|lllllll|}
D & A & A & B & C & D \\
A & D & A & D & B & C \\
A & A & D & C & D & B \\
\hline B & B & B & C & C & C \\
\hline D & D & D & A & A & A \\
\hline C & C & C & B & B & B \\
\hline
\end{array}
$$

In Betten (2019) we took the partitions $3+3$ for rows, columns, digits and transversals and started with the point tactical decomposition scheme A (figure PTD A). We applied a computer program, (Betten and Betten 2001, 2010; Betten and Braun 1992) which refines this point tactical decomposition scheme to a (or several) block tactical schemes. From each of them it goes on to point tactical schemes and so on. Either the process stops somewhere ore one gets one or more tactical decomposition schemes. 
The starting PTD A leads to TDO I and TDO II.

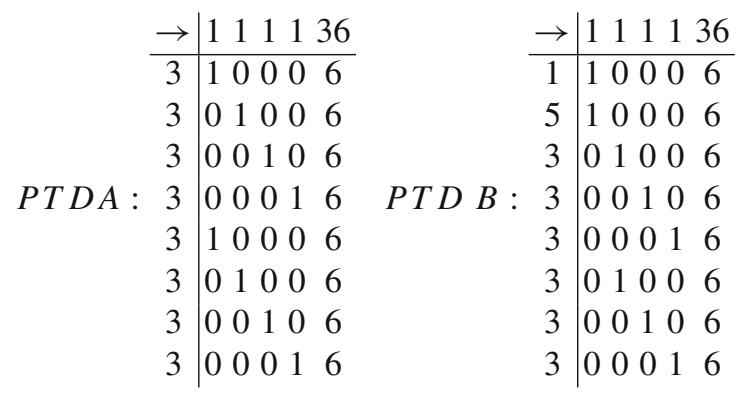

Improving this procedure we now begin with a finer starting decomposition B (figure PTD B), where the 6 rows are split into $1+5$. Then the refining process leads very quickly to only one tactical decomposition, the decomposition TDOII. After some permutation of blocks we get the decomposition of the theorem, noted in point tactical form.

The proof showed that among TDO I and TDO II only TDO II is possible, therefore

Theorem 2 A MOL(6) does not admit the tactical decomposition TDO I.

\section{The structure of TDO II}

\subsection{The invariant $\mu_{4}$}

The standard description of a MOL(6) is the following: Start with a $(6 \times 6)$-array of six rows and 6 columns $\{(r, c) ; r, c \in\{1,2,3,4,5,6\}\}$. In each cell $(r, c)$ is inserted a pair of numbers $(d, t), d, t \in\{1,2,3,4,5,6\}$, a digit $\mathrm{d}$ and a transversal $\mathrm{t}$ such that the conditions for a MOL(6) are fulfilled. Therefore one can view a MOL(6) as a 4-dimensional cube $\{(r, c, d, t) ; r, c, d, t \in\{1,2,3,4,5,6\}\} W_{6}$ with edge length 6 where 36 elements $M$ (the elements of the $\operatorname{MOL}(6))(r, c, d, t)$ are suitably distinguished.

The definition of $\mu_{4}$ :

Take a triple $1 \leq r_{1}<r_{2}<r_{3} \leq 6$ of rows, a triple $1 \leq c_{1}<c_{2}<c_{3} \leq 6$ of columns, a triple $1 \leq d_{1}<d_{2}<d_{3} \leq 6$ of digits and a triple $1 \leq t_{1}<t_{2}<t_{3} \leq 6$ of transversals. This defines a subcube $W_{3}$ of $W_{6}$ with edge length 3 . Let $\left|W_{3} \cap M\right|$ the number of elements of $\mathrm{M}$ contained in $W_{3}$, then we set

$$
\mu_{4}=\max \left\{\left|W_{3} \cap M\right|\right\}
$$

where $W_{3}$ varies over all $\left(\begin{array}{l}6 \\ 3\end{array}\right)^{4}$ subcubes with edge length 3 .

Theorem 3 Each MOL(6) has the invariant $\mu_{4}=6$. The subcube $W_{3}$ where the maximal number 6 is attained is unique. It has the quotient structure $\tilde{Q}$ which extends 
uniquely to the geometry $Q$ :

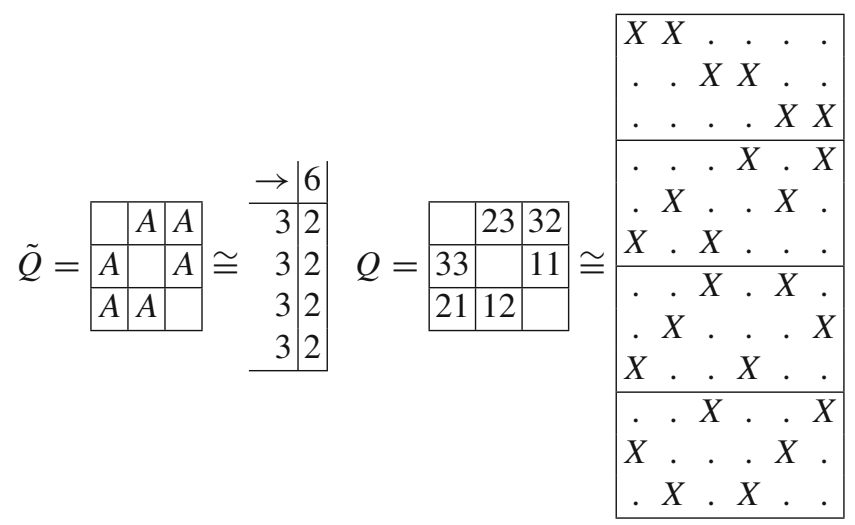

Proof The maximal number of elements in a subcube $W_{3}$ is given by the 4-blocks in the upper half of TDO II. This number is 6 , and therefore $\mu_{4}=6$.

\subsection{The parallel classes}

We consider 2-blocks on a set $\{1,2,3,4,5,6\}$ of 6 elements and we call 3 disjoint 2-blocks a parallel class. A parallel class is for instance $\{1,2\},\{3,4\},\{5,6\}$ :

$$
\begin{array}{|llllll|}
\hline & X & \cdot & \cdot & \cdot & \cdot \\
\cdot & \cdot & X & X & \cdot & \cdot \\
. & \cdot & \cdot & \cdot & X & X \\
\hline
\end{array}
$$

The geometry $Q$ in Theorem 3 consists of four disjoint parallel classes. We will show in the following that such a quadruple of parallel classes on a 6-element set is unique.

We recall the following theorem from (Betten 2019):

Theorem 4 There are up to isomorphism exactly two triples of parallel classes, namely $G:\{1,2\},\{3,4\},\{5,6\} ;\{2,3\},\{4,5\},\{6,1\} ;\{1,4\},\{2,5\},\{3,6\}$ and $N:\{1,2\},\{3,4\},\{5,6\} ;\{2,3\},\{4,5\},\{6,1\} ;\{1,5\},\{2,4\},\{3,6\}$.

There is exactly one quadruple of parallel classes:

$Q:\{1,2\},\{3,4\},\{5,6\} ;\{2,3\},\{4,5\},\{6,1\} ;\{1,5\},\{2,4\},\{3,6\}$; $\{1,3\},\{2,5\},\{4,6\}$.

The triple $G$ cannot be extended to the quadruple $Q$.

Proof There is obviously (up to isomorphism) only one pair of parallel classes, say $\{1,2\},\{3,4\},\{5,6\}$ and $\{2,3\},\{4,5\},\{6,1\}$.

A third class can consist of the three diagonal pairs

$$
G:\{1,4\},\{2,5\},\{3,6\} .
$$


If in the third class one pair is not diagonal then the other two pairs are unique and we get the type N. Now we construct a fourth class: For $G$ the remaining six 2-blocks form two triangles and from these one cannot choose three disjoint 2-blocks. For $N$ the remaining 6six 2-blocks form a 6-cycle which defines two disjoined parallel classes. So we get two quadruples of parallel classes which are obviously isomorphic.

In the following pictures we give the graphs of the two triples $\mathrm{G}$ and $\mathrm{N}$ and of the quadruple $\mathrm{Q}$ of parallel classes. We also give a description as parts of a latin square or part of an orthogonal pair.

The graphs:
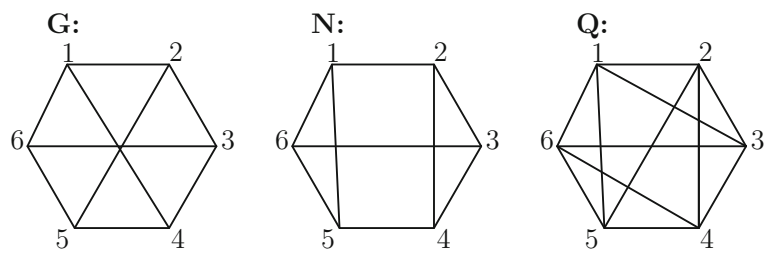

The partial squares:

$\mathrm{G}:$

\begin{tabular}{|l|l|l|}
\hline & 1 & 2 \\
\hline 1 & & 3 \\
\hline 2 & 3 &
\end{tabular}$\quad N:$\begin{tabular}{|l|l|l|}
\hline & 1 & 2 \\
\hline 2 & & 3 \\
\hline 1 & 3 & \\
\hline
\end{tabular}$\quad Q:$\begin{tabular}{|l|l|l|}
\hline & 11 & 22 \\
\hline 23 & & 31 \\
\hline 12 & 33 & \\
\hline
\end{tabular}$\cong$\begin{tabular}{|l|l|l|l|}
\hline & 23 & 32 \\
\hline 33 & & 11 \\
\hline 21 & 12 & \\
\hline
\end{tabular}

We call a triple of digits in $\mathrm{G}$ or in $\mathrm{N}$ tactical, if the three elements are in three different rows, three different columns and have three different values. The following is obvious:

Proposition 1 The six elements of $G$ can be splitted into two tactical triples. For $N$ this is not possible.

\subsection{Some geometries on nine and on 12 points}

Definition 1 An incidence geometry is called a TD3-geometry if it admits the tactical decomposition

$$
T D 3=\begin{array}{l|lll}
\rightarrow & 3 & 3 & 3 \\
\hline 3 & 1 & 1 & 0 \\
3 & 1 & 0 & 1 \\
3 & 0 & 1 & 1
\end{array}
$$

Theorem 5 There are, up to isomorphism, three TD3-geometries:

They have cycle distribution (3)(3)(3), (3)(6) resp. (9), if one draws the horizontal and vertical connections between flags.

Proof In order to generate the related geometries, we may choose by isomorphisms in five of the $3 \times 3$ squares the identity matrix, and only in the last (say right down) we have one of the six permutation matrices. If the three flags are on the main diagonal, then we get the distribution (3)(3)(3). If one element is on the diagonal, this leads to (3)(6) and if all three elements are disjoint to the diagonal, this is type (9). 

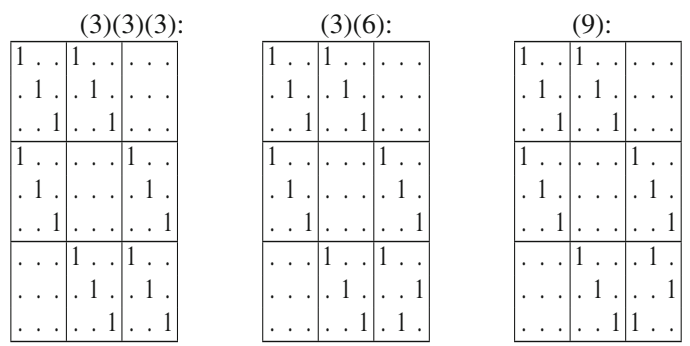

Definition 2 An incidence geometry is called a TD4-geometry if it admits the tactical decomposition

$$
T D 4=\begin{array}{c|ccc}
\rightarrow & 3 & 3 & 3 \\
\hline 3 & 1 & 1 & 0 \\
3 & 1 & 0 & 1 \\
3 & 0 & 1 & 1 \\
3 & 1 & 1 & 1
\end{array}
$$

The TD3-geometries with cycle distribution (3)(3)(3) or (9) can be extended to a TD4-geometry, but we show:

Theorem 6 The TD3-geometry with cycle distribution (3)(6) cannot be extended to a TD4-geometry.

Proof We take an isomorphism such that we get the triple $\{a, b, c\}$ left and the six cycle $\{1,2,3,4,5,6\}$ right:

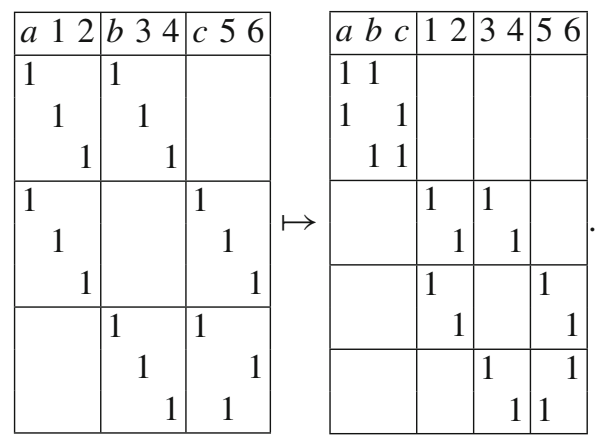

In order to extend according the last line of TD4 we need three disjoint lines of length 3 which have one element in $\{a, b, c\}$ and two elements in $\{1,2,3,4,5,6\}$. This means that we need a parallel class (three disjoint 2-blocks) on the set $\{1,2,3,4,5,6\}$. This is a six cycle $\{1,3,6,2,4,5\}$ which can be seen as two disjoint parallel classes. Furthermore the pairs $\{1,2\},,\{3,4\}$ and $\{5,6\}$ are not allowed, which we can interpret as a third parallel class. These three parallel classes have the type $\mathrm{G}$ and do not admit a fourth parallel class by Theorem 4 . So we get a contradiction. 
Remark: The derivation of TDO II and the proof of $\mu_{4}=6$ without computer can be found in Sect. 7.

\section{The non-existence of a MOL(6)}

\subsection{The distribution of the types A, B, C and D}

The 36 4-blocks of the MOL(6) split into 4 subsets: The sets A ,B, C, D of elements of type $\mathrm{A}, \mathrm{B}, \mathrm{C}$ and $\mathrm{D}$, respectively (we take the same names). Let $|A|,|B|,|C|,|D|$ be the numbers of elements of type $A, B, C, D$ in a row (or in a column), then

Proposition $2|A|=|D|$ and $|B|=|C|$

Proof In each row and each column there are three low and three high digits and also three low and three high transversals.

Examples for these four subsets are:
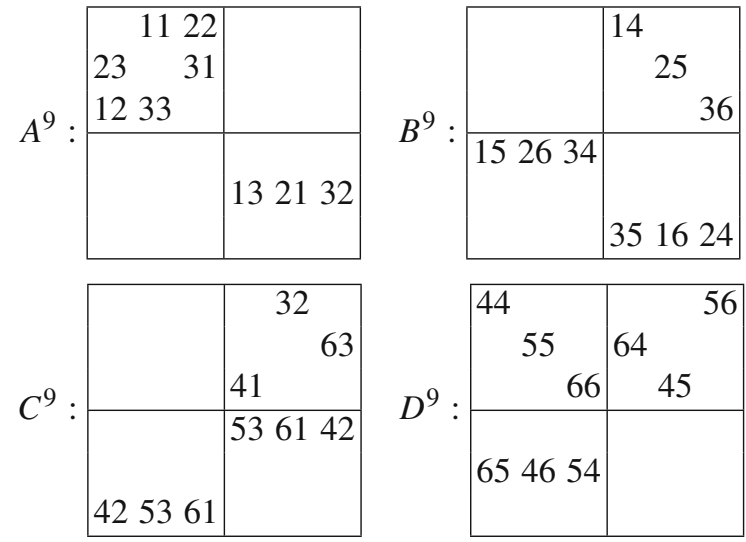

There is the following task: take for each of the four sets an isomorphism type which covers the same positions, and then try to put these four pieces together in order to get a MOL(6). We will not try this in general, but we will take suitable substructures of TDO II and show that they cannot be extended to a MOL(6). 


\subsection{A very short and simple non-existence proof}

Theorem 7 The following substructure of TDO II cannot be extended to a MOL(6):

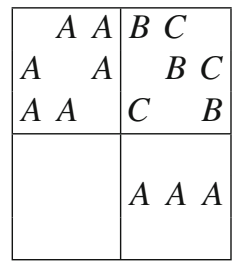

Proof a) The partial structure extends to

\begin{tabular}{|lrr|lll|}
\hline & 11 & 22 & $3 T$ & $H 3$ & \\
23 & & 31 & & $1 T$ & $H 2$ \\
12 & 33 & & $H 1$ & & $2 T$ \\
\hline & & & & \\
& & $A$ & $A$ & $A$ \\
& & & & \\
\hline
\end{tabular}

Proof The six elements A in row 1 to 3 , column 1 to 3 form the quadruple Q of parallel classes, which is unique up to isomorphism. So we can insert these six elements. Then in each of the three rows there is a low digit and a low transversal missing. Since the positions of $\mathrm{B}$ and $\mathrm{C}$ are given, we can insert these six elements uniquely.

(b) In row 5 there have to be inserted the three elements 13, 21, 32 in some permutation. This is not possible: the element 21 only fits into column 5, and also the element 32 fits only into column 5. This is a contradiction and the extension is not possible.

\subsection{The geometric background of the proof}

Though this is a very short proof for the non-existence of a MOL(6), it does not reveal the reason for the contradiction. For this we will now show the geometric background: We take a substructure of TDO II, shown in the following figure by the encircled elements. 


\begin{tabular}{|c|c|c|c|c|c|c|c|c|}
\hline & $\rightarrow$ & $\left|\begin{array}{llll}1 & 1 & 1 & 1\end{array}\right|$ & 0 & $J$ & & 3 & & \\
\hline row $1-3$ : & 3 & \begin{tabular}{llll|l}
1 & 0 & 0 & 0
\end{tabular} & (2) 1 & (1) (1) & & $\begin{array}{lll}0 & 0 & 0\end{array}$ & 0 & 0 \\
\hline $\operatorname{col} 1-3:$ & 3 & $\begin{array}{llll}0 & 1 & 0 & 0\end{array}$ & 21 & $\begin{array}{ll}0 & 0\end{array}$ & 0 & $\begin{array}{lll}1 & 1 & 1\end{array}$ & 0 & \\
\hline $\operatorname{dig} 1-3:$ & 3 & $\begin{array}{llll}0 & 0 & 1 & 0\end{array} \mid$ & (2) 0 & (1) 0 & 0 & 100 & (1) & \\
\hline$v 1-3:$ & 3 & $\begin{array}{lllll}0 & 0 & 0 & 1 \\
\end{array}$ & (2) 0 & 0 (1) & 0 & $\begin{array}{lll}0 & 1 & 0\end{array}$ & (1) & \\
\hline row 4: & 1 & $\begin{array}{lllll}1 & 0 & 0 & 0\end{array}$ & $\begin{array}{ll}0 & 0\end{array}$ & $\begin{array}{|ll|}0 & 0 \\
\end{array}$ & 0 & 300 & 0 & \\
\hline ow $5:$ & 1 & $\begin{array}{lllll}1 & 0 & 0 & 0\end{array}$ & $\begin{array}{ll}0 & 0 \\
\end{array}$ & $\begin{array}{ll}0 & 0 \\
\end{array}$ & 0 & $\begin{array}{lll}0 & 3 & 0\end{array}$ & 0 & \\
\hline row 6 : & 1 & 10000 & 00 & $\begin{array}{ll}0 & 0 \\
\end{array}$ & 0 & $\begin{array}{lll}0 & 0 & 3\end{array}$ & 3 & \\
\hline$l 4-6:$ & 3 & \begin{tabular}{llll|l}
0 & 1 & 0 & 0
\end{tabular} & 0 & (1) (1) & 1 & $\begin{array}{lll}0 & 0 & 0\end{array}$ & (1) & \\
\hline $\operatorname{dig} 4-6:$ & 3 & $\begin{array}{lllll}0 & 0 & 1 & 0\end{array}$ & $\begin{array}{ll}0 & 1\end{array}$ & $\begin{array}{ll}0 & 1\end{array}$ & 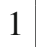 & $\begin{array}{lll}0 & 1 & 1\end{array}$ & 0 & \\
\hline \multirow[t]{2}{*}{$\operatorname{trsv} 4-6:$} & 3 & $\begin{array}{llll}0 & 0 & 0 & 1\end{array}$ & $\begin{array}{ll}0 & 1 \\
\end{array}$ & 10 & 1 & $\begin{array}{lll}1 & 0 & 1 \\
\end{array}$ & 0 & 1 \\
\hline & & & & $B C$ & & $B C D$ & & \\
\hline
\end{tabular}

This substructure has the following tactical decomposition:

$$
T D 5=\begin{array}{c|c||ccc|}
\rightarrow & 6 & 3 & 3 & 3 \\
\hline 3 & 2 & 1 & 1 & 0 \\
3 & 2 & 1 & 0 & 1 \\
3 & 2 & 0 & 1 & 1 \\
\hline 3 & 0 & 1 & 1 & 1 \\
& A & C & A
\end{array} \mid
$$

This tactical decomposition contains the tactical decomposition TD3 (square upper right), and we show

Proposition 3 The TD3 contained in TD5 has cycle type (3)(6).

Proof The first column of TD5 is a triple of parallel classes on a set of 6 elements. Since this is contained in a quadruple of parallel classes (see TDO II), it has the type $\mathrm{N}$ by Theorem 4. Up to isomorphism we can take

$$
\begin{array}{|rrr|}
\hline & .3 & .2 \\
.1 & & .3 \\
.2 & .1 & \\
\hline
\end{array}
$$

as part of a latin square. Here the notation ".3" means that the digit is unknown and the transversal is 3 , similarly ". 1 " and ".2".

We give a presentation as a geometry on 9 variables, and 6 3-blocks and add the missing 2-blocks. These nine 2-blocks define the TD3-geometry, and checking the 
cycles, we find the cycle distribution (3)(6):

\begin{tabular}{|c|c|c|c|}
\hline $\begin{array}{llllll}1 & 1 & . & . & . & . \\
\cdot & . & 1 & 1 & . & . \\
. & . & . & . & 1 & 1\end{array}$ & $\begin{array}{lll}1 & . & . \\
. & 1 & . \\
. & . & 1\end{array}$ & $\begin{array}{lll}1 & . & . \\
. & 1 & . \\
. & . & 1\end{array}$ & . \\
\hline $\begin{array}{llllll} & . & 1 & . & 1 & \\
1 & . & . & . & . & 1 \\
. & 1 & . & 1 & . & . \\
\end{array}$ & $\begin{array}{lll} & . & . \\
. & 1 & . \\
. & . & 1\end{array}$ & 5 & $\begin{array}{ll} & . \\
. & 1 \\
. & . \\
\end{array}$ \\
\hline $\begin{array}{llllll} & . & . & 1 & & . \\
. & 1 & . & . & 1 & \\
. & . & 1 & . & . & 1\end{array} \mid$ & & $\begin{array}{l}. \quad .1 \\
. \\
1\end{array}$ & $\begin{array}{l}1 . \\
. \\
. \\
.\end{array}$ \\
\hline
\end{tabular}

Theorem 8 No MOL(6) exists.

Proof We prove that TD5 cannot be extended to a geometry: Starting with the first column and using Proposition 3, it follows that the TD3-geometry has type (3)(6). By Theorem 6 this geometry cannot be extended to the TD4-geometry, a contradiction.

Remark: The main geometric reason for the non-solvability of the Euler problem is Theorem 4: a triple of parallel classes of type $\mathrm{G}$ cannot be extended to a quadruple of parallel classes. We used this fact even twice: First in Proposition 3 to show that the TD3-geometry has cycle type (3)(6), and then in Theorem 6 to prove that an extension to the TD4-geometry is not possible.

\section{The first three rows}

When one arranges the 36 officers, one would like to do this row by row. So we will describe in the following the first three rows. We take the TDO II in the form:

$$
T D O I I \cong \begin{array}{lll|lll|}
D & A & A & D & B & C \\
A & D & A & C & D & B \\
A & A & D & B & C & D \\
\hline D & D & D & A & A & A \\
B & B & B & C & C & C \\
C & C & C & B & B & B \\
\hline
\end{array} \text {. }
$$

Proposition 4 The first three rows of a $M O L(6)$ are

\begin{tabular}{|lll|lll|}
\hline 44 & 11 & 22 & 56 & 35 & 63 \\
23 & 55 & 31 & 42 & 64 & 16 \\
12 & 33 & 66 & 24 & 51 & 45 \\
\hline
\end{tabular}


Proof Up to isomorphism we can take the first three columns as given in the proposition. Now we add the three elements $\mathrm{D}$ at the right hand side:

\begin{tabular}{|c|c|c|c|c|}
\hline 441122 & & & 441122 & \\
\hline 235531 & 64 & or & 235531 & 46 \\
\hline 123366 & 45 & & 123366 & 54 \\
\hline
\end{tabular}

Using the positions of the three elements B and C, we can fill in the first case uniquely. In the second case we get the element 14 on position 26 , a contradiction to the element 54 on position 36.

Proposition 5 The row triple of Proposition 4 cannot be contained in a MOL(6).

Proof In row 2 and 3, columns 1,4,6 there are the elements

234216

122445

Here the digits form a three-cycle and need six different transversals (Proposition 7b). But there are two equal transversals .2, a contradiction.

Though this is already a non-existence proof for a MOL(6), we are interested in the possible extensions of the triple of rows.

Proposition 6 The first three rows extend uniquely to the $(6 \times 6)$-array

\begin{tabular}{|lll|lll|}
\hline 44 & 11 & 22 & 56 & 35 & 63 \\
23 & 55 & 31 & 42 & 64 & 16 \\
12 & 33 & 66 & 24 & 51 & 45 \\
\hline 65 & 46 & 54 & 33 & 12 & 21 \\
36 & 24 & 15 & 61 & 43 & 52 \\
51 & 62 & 43 & 15 & 26 & 34 \\
\hline
\end{tabular}

Proof Columns 1 to 3:

We put the three elements $\mathrm{D}$ into row 4 and the three columns follow uniquely (using the types $\mathrm{A}, \mathrm{B}, \mathrm{C}, \mathrm{D})$ of the TDO II).

Columns 4 to 6 :

The high digits in row 5 and the high transversals in row 6 follow from their column. In order to determine their low partners (transversal resp. digit), we check the actual column and the elements in rows 1 to 3 . This determines the 6 elements in row 5 and 6 uniquely. Take for example .4 on position 66 . Then in column 6 there is a low digit 1 , and 1 cannot be taken. Furthermore there is a 24 on row 3, therefore the digit 2 is forbidden. It remains the low digit 3 and we get the element $.4=34$. In this way we can fill row 5 and 6 , and the three elements $A=t d$ on row 4 follow by their column.

Theorem 9 This $(6 \times 6)$-array is not a MOL(6), and therefore a MOL(6) does not exist. 
Proof The pairs (digit/transversal) 12, 15, 24, 33, 43, 51 occur twice, and the pairs $13,14,25,32,41,53$ are missing. This is a contradiction since in a MOL(6) each of the 36 pairs occurs exactly once.

\section{The four latin squares contained in TDO II}

Each MOL(6) has six rows R, six columns C, six digits D and six transversals T. It contains four latin squares of order 6, which are the restrictions to $R \cup C \cup D$, $R \cup C \cup T, R \cup D \cup T$ and $C \cup D \cup T$. If one deletes from the tactical decomposition TDO II in Theorem 2 the six rows (lines 1 to 3 and lines 13, 14, 15), then one gets a tactical decomposition for the latin square on $C \cup D \cup T$ :

Tactical decomposition of type A:
\begin{tabular}{llllllllllllllll}
$\rightarrow \rightarrow$ & 1 & 1 & 1 & 1 & 6 & 3 & 3 & 3 & 3 & 3 & 3 & 3 & 3 & 3 & 3 \\
\hline $3 \mid$ & 1 & 0 & 0 & 0 & 2 & 1 & 1 & 1 & 1 & 0 & 0 & 0 & 0 & 0 & 0 \\
$3 \mid$ & 0 & 1 & 0 & 0 & 2 & 1 & 0 & 0 & 0 & 1 & 1 & 1 & 0 & 0 & 0 \\
$3 \mid$ & 0 & 0 & 1 & 0 & 2 & 0 & 1 & 0 & 0 & 1 & 0 & 0 & 1 & 1 & 0 \\
\hline $3 \mid$ & 1 & 0 & 0 & 0 & 0 & 0 & 0 & 0 & 0 & 1 & 1 & 1 & 1 & 1 & 1 \\
$3 \mid$ & 0 & 1 & 0 & 0 & 0 & 0 & 1 & 1 & 1 & 0 & 0 & 0 & 1 & 1 & 1 \\
$3 \mid$ & 0 & 0 & 1 & 0 & 0 & 1 & 0 & 1 & 1 & 0 & 1 & 1 & 0 & 0 & 1
\end{tabular}

Theorem 10 The tactical decomposition of type A does not generate a latin square $L Q(6)$ which is embedded in a MOL(6).

Proof Define

$$
N \cong \begin{array}{lll}
1 & 4 & 5 \\
4 & 2 & 6 \\
6 & 5 & 3
\end{array} \text { and } G \cong \begin{array}{lll}
1 & 4 & 5 \\
4 & 2 & 6 \\
5 & 6 & 3
\end{array}
$$

The latin square generated by the tactical decomposition A has a $3 \times 3$ subsquare (left above) with type G or N. Since the latin square is a subsquare of a MOL(6) the type can only be $\mathrm{N}$ by Theorem 4 . The tactical decomposition A shows that the three $3 \times 3$ subsquares right above, left down and right down have all three the type $\mathrm{G}$ by Proposition 1 . Now starting with the $(3 \times 3)$-square left above and extending according to the decomposition A, we get directly (without distinction of cases) the following partial latin square:

\begin{tabular}{|rrr|lll|}
\hline 4 & 1 & 2 & 3 & 6 & 5 \\
2 & 5 & 3 & 6 & 1 & 4 \\
1 & 3 & 6 & 5 & 4 & 2 \\
\hline 3 & 6 & 5 & 4 & \\
6 & 2 & 4 & \multicolumn{2}{|c|}{5} \\
5 & 4 & 1 & & \\
\hline
\end{tabular}


Now we have to fill in the missing two triples $\{1,2,3\}$ into the box right down. This is possible in a unique way, but only with type $\mathrm{N}$. This contradicts the decomposition A, where we need the type G.

\section{Two partial substructures}

In Betten (1983) there were derived three different shapes (A), (B) and (C) for latin squares of order 6 . It was proved that squares of shape (A) and (B) have no or at most four transversals, therefore they cannot have an orthogonal square. So only squares of shape $\mathrm{C}$ have to be considered for the problem of Euler and Tarry. Let $d \in\{1,2,3\}$ and $H \in\{4,5,6\}$ then we give in the following figure the shape $\mathrm{C}$ ) and a special example:

Type C: \begin{tabular}{|llll|lll|}
\hline$d$ & $d$ & $d$ & $H$ & $H$ & $H$ \\
$d$ & $d$ & $d$ & $H$ & $H$ & $H$ \\
\hline$H$ & $H$ & $H$ & $d$ & $d$ & $d$ \\
\hline$d$ & $H$ & $H$ & $H$ & $d$ & $d$ \\
$H$ & $d$ & $H$ & $d$ & $H$ & $d$ \\
$H$ & $H$ & $d$ & $d$ & $d$ & $H$ \\
\hline
\end{tabular} \mid \begin{tabular}{|lllllll|}
2 & 3 & 1 & 5 & 6 & 4 \\
3 & 1 & 2 & 6 & 4 & 5 \\
\hline 4 & 6 & 5 & 1 & 2 & 3 \\
\hline 1 & 5 & 6 & 4 & 3 & 2 \\
5 & 2 & 4 & 3 & 5 & 1 \\
6 & 4 & 3 & 2 & 1 & 6 \\
\hline
\end{tabular}.

In the square left down (rows 4 to 6 , column 1 to 3 ) there are three elements $\mathrm{d}$ on the diagonal. We call them the distinguished elements. Similarly, we call the three elements $\mathrm{H}$ in the square right down (rows 4 to 6 , columns 4 to 6 ) the distinguished elements in this square. There are the following conditions for the transversals in Case C, see [Betten (1983), Lemma p. 425]:

Proposition 7 (a) The three elements with low digits in the $(3 \times 3)$-subsquare left down have three different transversals. The same transversals occur on the three distinguished digits right down.

(b) In each of the two $(2 \times 3)$-rectangles in the first two rows there are six different transversals.

Theorem 11 (a) Each MOL(6) contains one of the following two partial structures:

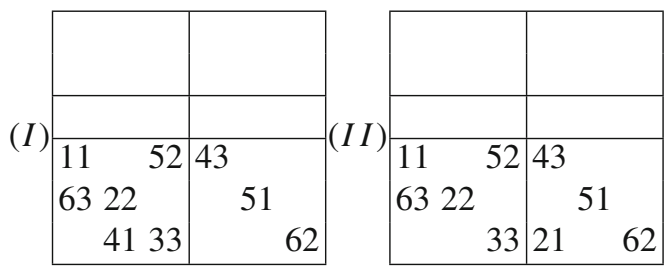

(b) These two cases correspond to $\mu_{4}=6$ and $\mu_{4}=5$ respectively. 
(c) The two cases extend to the following substructures of a MOL(6):

$(I) \mid$\begin{tabular}{lll|lll|}
\hline$A$ & $A$ & $A$ & $D$ & $D$ & $D$ \\
$B$ & $B$ & $B$ & $C$ & $C$ & $C$ \\
\hline$D$ & $D$ & $D$ & $A$ & $A$ & $A$ \\
\hline$A$ & $D$ & $C$ & $C$ & $B$ & $B$ \\
$C$ & $A$ & $D$ & $B$ & $C$ & $B$ \\
$D$ & $C$ & $A$ & $B$ & $B$ & $C$ \\
\hline
\end{tabular}

$(I I) \mid$\begin{tabular}{lll|lll|}
$A$ & $A$ & $B$ & $D$ & $D$ & $C$ \\
$B$ & $A$ & $B$ & $D$ & $C$ & $C$ \\
\hline$D$ & $D$ & $C$ & $A$ & $A$ & $B$ \\
\hline$A$ & $D$ & $C$ & $C$ & $B$ & $B$ \\
$C$ & $A$ & $D$ & $B$ & $C$ & $B$ \\
$D$ & $D$ & $A$ & $A$ & $B$ & $C$ \\
\hline
\end{tabular}

Proof (a) By Proposition 7(a) there is up to isomorphism the substructure

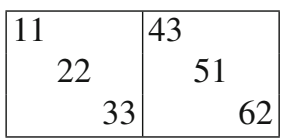

In each of the three rows, there is a low transversal missing. These three elements can be on one side, say columns 1 to 3, or up to interchanging left and right, two elements are left (column 1 to 3 ) and one element on the right side (columns 4 to 6).

(b) In case I, the 6 missing elements on the right side have low digits and high transversals, therefore we get $\mu_{4}=6$. In case II there are five elements with low digits and high transversals, and it follows $\mu_{4}=5$.

(c) In each of the $(2 \times 3)$-rectangles in the first two rows, there are 3 low transversals by Proposition 7(b). Therefore we get the extensions

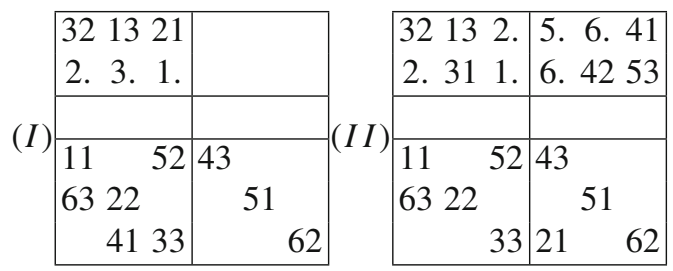

In the last three rows we know all digits (low or high) since the latin squares made of digits are of shape $\mathrm{C}$. We also know that all missing transversals are high since the low transversals are spent. Since in all rows and all columns there are three digits d, three digits $\mathrm{H}$, three transversals $\mathrm{t}$ and three transversals $\mathrm{T}$, we get uniquely

$(I)$\begin{tabular}{|ccc|ccc}
32 & 13 & 21 & $H T$ & $H T$ & $H T$ \\
$2 T$ & $3 T$ & $1 T$ & $H t$ & $H t$ & $H t$ \\
\hline$H T$ & $H T$ & $H T$ & $d t$ & $d t$ & $d t$ \\
\hline 11 & $H T$ & 52 & 43 & $d T$ & $d T$ \\
63 & 22 & $H T$ & $d T$ & 51 & $d T$ \\
$H T$ & 41 & 33 & $d T$ & $d T$ & 62
\end{tabular}$\quad(I I)$\begin{tabular}{|ccc|ccc|}
32 \\
$2 T$ & 31 & $1 T$ & $6 T$ & 42 & 53 \\
\hline$H T$ & $H T$ & $6 t$ & $d t$ & $d t$ & $d T$ \\
\hline 11 & $H T$ & 52 & 43 & $d T$ & $d T$ \\
63 & 22 & $4 T$ & $d T$ & 51 & $d T$ \\
$H T$ & $H T$ & 33 & 21 & $d T$ & 62 \\
\hline
\end{tabular}

Using the notations A, B, C and D we get the theorem.

Theorem 12 (a) Case II does not exist and 
(b) Case I is isomorphic to TDO II.

Proof (a) We extract lines 3 and 6:

$$
\begin{array}{|lll|lll|}
\hline H T & H T & 6 t & d t & d t & d T \\
\hline H T & H T & 33 & 21 & d T & 62 \\
\hline
\end{array}
$$

Since the high digits 6 are already spent, we get in the first two columns the 2-squares $\left(\begin{array}{ll}4 & 5 \\ 5 & 4\end{array}\right)$ or $\left(\begin{array}{ll}5 & 4 \\ 4 & 5\end{array}\right)$. These four elements have high transversals and since there are only three values for high transversals, we get a contradiction.

(b) In Case (I) we interchange $A \leftrightarrow B$ and $C \leftrightarrow D$ and interchange columns 1-3 with columns $4-6$. Then after a suitable permutation of the rows we get TDO II.

Remark: In this way we derived TDO II and proved $\mu_{4}=6$ without use of the computer.

\section{The automorphism group of a MOL(6)}

Proposition 8 The following figure describes a parallelism on six points. This parallelism has automorphism group $S_{5}$ acting as symmetric group on the set of five parallel classes.

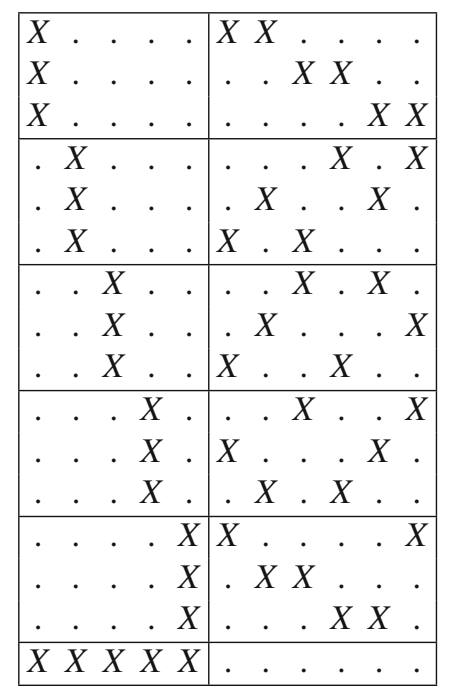

We take as six-element set the six columns describing five-blocks. There are horizontally five parallel classes $2+2+2$. Since the two-blocks in all parallel classes exhaust the 15 pairs of the 6 columns we have a so called parallelism (also called a packing). For each parallel class the two-lines are extended by a point "at infinity" or the direction, so we get three lines of length three intersecting in this point. We define a new line of length 5 , incident with the five points at infinity called the line at infinity (or the 
improper line). Then we get a linear space with 11 points and the line length distribution $5^{1}, 3^{15}$. This linear space is unique and has the symmetric group $S_{5}$ as group of collineations, see also the list [Betten and Betten (1999), p. 23]. The collineation group $S_{5}$ acts as symmetric group on the line at infinity, but it acts also transitively on the set of six 5-blocks. Recall that $S_{6}$ has two types of subgroups $S_{5}$ : One fixing a point and acting on the rest as $S_{5}$, the other acting transitively on the six-set. This second action cannot be seen well, but the parallelism is a nice model for it.

Proposition 9 The automorphism group of $Q$ is the symmetric group $S_{4}$.

Proof The parallelism on six points has automorphism group $S_{5}$ acting as symmetric group on the set of five parallel classes. Fixing one class one gets the quadruple Q of parallel classes with automorphism group $S_{4}$.

We showed that a MOL(6) has the substructure Q and the max number $\mu_{4}=6$. Conversely, we now suppose that there is a substructure $\mathrm{Q}$ and we state:

Theorem 13 Each MOL(6) with a substructure $Q$ has the following form:

MOL(6)withsubstructure $Q$ :

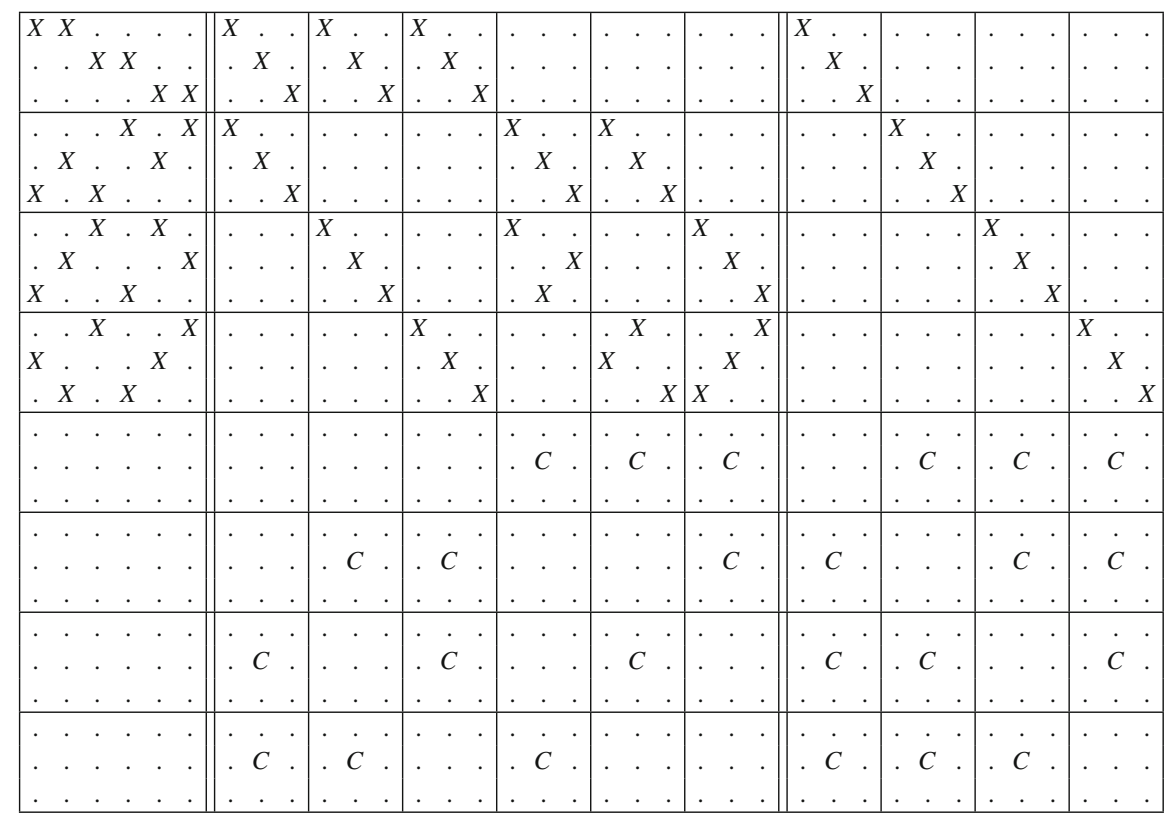

Here the upper part (rows 1 to 12$)$ is given explicitely. Each of the $(3 \times 3)$ cells in the lower part (rows 13 to 24) is isomorphic to

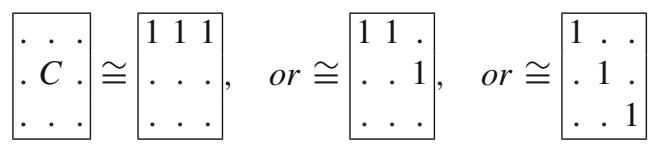


Proof We first construct the upper 12 lines. Since Q is - up to isomorphism - unique, we can put in the six 4-blocks of Q. Dually Q consists of four parallel classes $(2+2+2)$ on six elements, the six 4-blocks. Each pair of parallel classes defines six vertical pairs of flags, and we need three more vertical pairs since we construct a linear space on 12 points. Since there are six pairs of parallel classes we get six triples of vertical pairs, which we add. Counting flags in their 12 lines, we see that in each line there is one more flag, and this gives 12 blocks having one flag in the upper part.

We now construct the 12 lines in the lower part: Since we construct a linear space on 24 points, each pair of vertical flags in the upper part defines uniquely two vertical flags in the lower part. And also the 12 single flags in the upper part can uniquely be extended to a 4-block by adding three suitable flags in the lower part. Note however that the lower part is only block tactical, which gives the various types $\mathrm{C}$ of $(3 \times 3)$-cells.

Theorem 14 Each MOL(6) with a subgeometry Q has the automorphism group $\Gamma=S_{4}$ where $S_{4}$ is the symmetric group on the four parallel classes. The group $\Gamma$ acts on the decomposition scheme with eight orbits, given by the separating double lines in the following figure:

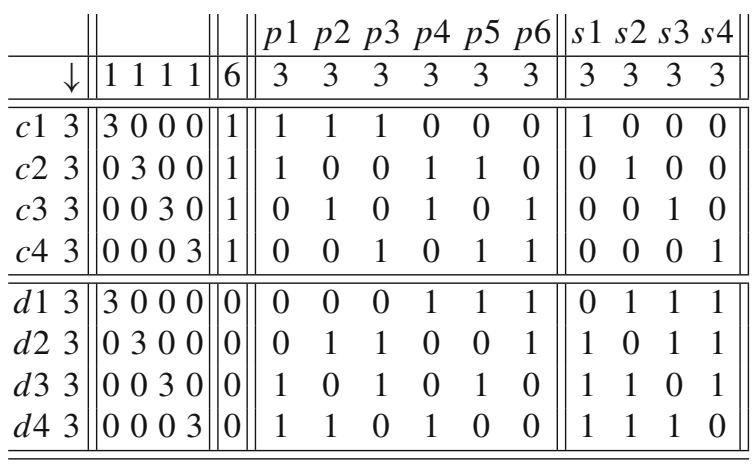

Proof (a) The geometry Q has automorphism group $S_{4}$ and extends uniquely with group to the first 12 lines of the MOL(6) and on the set of 12 blocks with one flag in the upper part. Therefore we know the upper part (first 12 lines) of the geometry explicitly- the geometry and the action of $\Gamma$ on it.

(b) We now study the lower part (rows 5 to 8) of the decomposition resp. Lines 13 to 24 of the geometry. We denote the first 4 rows of the decomposition by $c 1, c 2, c 3, c 4$, the four parallel classes, and rows 5 to 8 by $d 1, d 2, d 3, d 4$. Since the 3-block of ci combines with the 3-block of di to a 6-block of the geometry, $i=1,2,3,4$, the action on $\{c 1, c 2, c 3, c 4\}$ is the same as the action on $\{d 1, d 2, d 3, d 4\}$. We denote further the 6 triples of 2-blocks by $p 1, p 2, p 3, p 4, p 5, p 6$ and the 4 triples of 1-blocks by $s 1, s 2, s 3, s 4$.

The group $\Gamma$ acts transitively on the set of four parallel classes and also transitively on the set of six pairs of parallel classes, and therefore it is transitive on the set of six columns $p 1, p 2, \ldots, p 6$ and also transitive on the four columns $s 1, s 2, s 3, s 4$. Now take the involution in $S_{4}$ which interchanges c1 and $\mathrm{c} 2$ and fixes c3 and c4. Then it fixes also p1 and p6 and interchanges $\mathrm{d} 1$ and $\mathrm{d} 2$. It follows that the following vertical 
pairs of 3-cells are interchanged: (c1,p1) with (c2,p1), (d1,p6) with (d2,p6) and (d1,s4) with $(\mathrm{d} 2, \mathrm{~s} 4)$. Therefore the transitivity of $S_{4}$ on the four regions is proved.

Theorem 15 All $(3 \times 3)$-cells in the lower part of Theorem 13 are tactical, i.,e. isomorphic to $P=\begin{array}{lll}1 & . & . \\ . & 1 & . \\ . & . & 1\end{array}$.

Proof The involution $\alpha=\left(\begin{array}{l}0 \\ \text { 2) }\end{array}\right)\left(\begin{array}{l}3 \\ 5\end{array}\right)\left(\begin{array}{l}6 \\ 11\end{array}\right)\left(\begin{array}{l}7 \\ 10\end{array}\right)(8$ 9) $\in \Gamma$ fixes each block in p6. These three blocks extend to $\mathrm{d} 1$ and $\mathrm{d} 2$ and $\alpha$ interchanges $\mathrm{d} 1$ with $\mathrm{d} 2$. Therefore the three flags in $(\mathrm{d} 1, \mathrm{p} 2)$ and in $(\mathrm{d} 2, \mathrm{p} 2)$ are tactical since two points are joined by only one block.

By transitivity, all (occupied) $(3 \times 3)$-cells in the lower part are tactical. The three cells (c4,p5), (c45,p6), (c4,s4) are in the upper part and tactical. The two cells (c2,p5), $(\mathrm{c} 2, \mathrm{p} 6)$ are tactical, therefore also $(\mathrm{c} 2, \mathrm{~s} 4)$ is tactical. By transitivity all cells in the lower part are tactical.

Remark: This means that the decomposition is isomorphic to TDO I, but TDO I does not exist by Theorem 2 .

\section{Disjoint 4-blocks}

In Betten (2021) we proved the following theorem: The maximal number of pairwise disjoint four-blocks in a MOL(6) is 3. We apply this theorem and show

Theorem 16 In TDO II, there are four pairwise disjoint four-blocks.

\section{Proof}

$$
T \text { DO II } \cong \begin{array}{lll|lll|}
\hline \text { D) } & A & A & D & B & C \\
A & \text { D } & A & C & D & B \\
A & A & \text { D } & B & C & D \\
\hline D & D & D & A & A & A \\
B & B & B & C & C & C \\
C & C & C & B & B & B \\
\hline
\end{array} .
$$

The four encircled elements in TDO II correspond to four pairwise disjoint 4-blocks in the tactical decomposition: each pair has different rows, different columns, different digits and different transversals.

Remark: If we already know TDO II and we use the theorem of disjoint blocks, we get a direct contradiction to the existence of a MOL(6) without further work.

Funding Open Access funding enabled and organized by Projekt DEAL.

Open Access This article is licensed under a Creative Commons Attribution 4.0 International License, which permits use, sharing, adaptation, distribution and reproduction in any medium or format, as long as you give appropriate credit to the original author(s) and the source, provide a link to the Creative Commons licence, 
and indicate if changes were made. The images or other third party material in this article are included in the article's Creative Commons licence, unless indicated otherwise in a credit line to the material. If material is not included in the article's Creative Commons licence and your intended use is not permitted by statutory regulation or exceeds the permitted use, you will need to obtain permission directly from the copyright holder. To view a copy of this licence, visit http://creativecommons.org/licenses/by/4.0/.

\section{References}

Beth, Th., Jungnickel, D., Lenz, H.: Design Theory. Bibl. Inst. Mannheim, Wien (1985)

Betten, A., and Betten, D.: Proper linear spaces on 18 points. In: Betten, A., et al. (eds) Proceedings European Conference of Algebraic Combinatorics Application Gössweinstein, Sept. 12-19, 1999, Springer , 40-54 (2001)

Betten, D.: Zum Satz von Euler-Tarry. Der mathematische und naturwissenschaftliche Unterricht 36, 449453 (1983)

Betten, D.: Die 12 lateinischen Quadrate der Ordnung 6. Mitteilungen Math. Sem. Giessen 136, 181-188 (1984)

Betten, D.: The Reye configuration in a MOL(6). Mitt. Math. Ges. Hamburg 39, 59-76 (2019)

Betten, D.: Disjoint blocks in a MOL(6). Res. Math. 76, 9 (2021)

Betten, D.: The problem of Euler/Tarry revisited. Beitr. Algebra Geom. 62, 815-821 (2021). https://doi. org/10.1007/s13366-020-00547-y

Betten, A., Betten, D.: Regular linear spaces. Beiträge zur Alg. und Geom. 38, 111-124 (1997)

Betten, A., Betten, D.: Linear spaces with at most 12 points. J. Comb. Des. 7, 119-145 (1999)

Betten, A., Betten, D.: There is no Drake/Larson linear space on 30 points. J. Comb. Des. 18, 48-70 (2010)

Betten, D., Braun, M.: A tactical decomposition for incidence structures. Ann. Discr. Math. 52, 37-43 (1992)

Colbourne, C.J., Dinitz, J.H.: The CRC Handbook of Combinatorial Designs. CRC Press, Boca Raton (1996)

Denes, J., Keedwell, A.D.: Latin Squares and their Applications. English University Press, London (1974)

Euler, L.: Recherches sur une nouvelle espece des quarres magiques, Leonardi Euleri opera, ser. I, vol. 7, pp. 291-392, Leipzig, Brlin: Teubner 1923 (1782)

Fisher, R.A., Yates, F.: The 6x6 Latin squares. Proc. Camb. Philos. Soc. 30, 492-507 (1934)

Stinson, D.R.: A short proof of the Nonexistence of a Pair of Orthogonal Latin Squares of Order Six. J. Comb. Theory A 36, 373-376 (1984)

M.G. Tarry: Le probleme des 36 officiers, Comptes rendus Ass. Franc. Sci. Nat. 1 (1900), 2, 170-203 (1901)

Publisher's Note Springer Nature remains neutral with regard to jurisdictional claims in published maps and institutional affiliations. 